\title{
Evaluation of different surgical modalities for coronary reconstruction of diffusely diseased left anterior descending artery
}

\author{
Mohamed H. Elsayed ${ }^{1}$, Wael M. Hassanein'1, Samir A. Keshk¹, Mamdouh Zidan², Waheed G. Etman \\ 'Department of Cardiothoracic Surgery, University of Alexandria, Alexandria 21111, Egypt. \\ ${ }^{2}$ Department of Radiology, University of Alexandria, Alexandria 21111, Egypt.
}

Correspondence to: Dr. Mohamed H. Elsayed, Department of Cardiothoracic Surgery, University of Alexandria, Champollion Street, Khartoum Square, Azarita, Alexandria 21111, Egypt.E-mail: moh.hassanein@gmail.com

\begin{abstract}
How to cite this article: Elsayed MH, Hassanein WM, Keshk SA, Zidan M, Etman WG. Evaluation of different surgical modalities for coronary reconstruction of diffusely diseased left anterior descending artery. Vessel Plus 2018;2:39. http://dx.doi.org/10.20517/2574-1209.2018.65
\end{abstract}

Received: 13 Sep 2018 First Decision: 26 Oct 2018 Revised: 30 Oct 2018 Accepted: 1 Nov 2018 Published: 12 Nov 2018

Science Editor: Mario F. L. Gaudino Copy Editor: Cui Yu Production Editor: Zhong-Yu Guo

\begin{abstract}
Aim: Endarterectomy has been shown to be an effective adjunct in treating diffusely diseased coronary arteries. Reconstruction of endarterectomized coronaries has been done by various techniques. We compare early results of left internal mammary artery (LIMA) patch to saphenous vein patch in left anterior descending artery (LAD) reconstruction.
\end{abstract}

Methods: We prospectively followed 30 patients with diffusely diseased LAD from January 2016 to January 2018. Patients were followed up clinically, by echocardiogram and CT coronary angiography.

Results: Twenty-seven patients were males (90\%). The mean age was $59.23 \pm 7.98$. Twenty-two patients (73.3\%) had a LIMA onlay patch. The mean length of patch reconstruction was greater in the saphenous vein group than LIMA group $(8.31 \pm 1.16 \mathrm{~cm}$ vs. $5.64 \pm 0.73 \mathrm{~cm}, P<0.001)$. Postoperative myocardial infarction occurred in 1 patient from the LIMA group (4.5\%) and 1 patient in the saphenous vein patch group (12.5\%). Operative mortality occurred in 1 patient belonging to the LIMA group. Mean time of follow up was $17.59 \pm 6.34$ months. CT coronary angiography showed a patency rate of $93.1 \%$.

Conclusion: Results of reconstruction by LIMA and saphenous vein patch are comparable in short-term follow up.

Keywords: Endarterectomy, reconstruction, left internal mammary artery patch 


\section{INTRODUCTION}

The progressive application of percutaneous coronary interventions (PCI) to achieve myocardial revascularization has contributed to the referral of patients with distinctly less attractive anatomic substrates for surgery. Surgical candidates now are usually from an older age group, have more severe coronary lesions and suffer from multiple comorbidities. Of these perhaps diffuse coronary disease is one of the most troublesome situations the surgeon has to deal with.

A conventional anastomosis placed distally in a diffusely diseased vessel such as the left anterior descending artery (LAD) may leave a large area of myocardium supplied by large side branches unrevascularized, which defies the principle of coronary artery bypass grafting (CABG) aiming at complete revascularization ${ }^{[1]}$. Hence endarterectomy has been revisited as an adjunct to conventional CABG in such cases.

Coronary endarterectomy is done by different surgical techniques, mainly open and closed. However in principle, they all entail removal of the atherosclerotic plaque or calcified core of the coronary vessel creating a neo-vascular bed which can be revascularized using one of the standard arterial or venous conduits.

Since Bailey's first coronary endarterectomy ${ }^{[2]}$, a lot has changed, namely the use of cardiopulmonary bypass, pharmacological support, and the growing experience of cardiac surgeons. In the current era results have changed significantly from earlier days where controversial debates were held about coronary endarterectomy due to its morbidity and mortality mainly perioperative myocardial infarction $(\mathrm{MI})^{[3]}$. It is therefore important to focus on the current results and proper indications for selecting this technique.

In this study we evaluate the outcome of different surgical modalities for coronary reconstruction in diffusely diseased LAD.

\section{METHODS}

Thirty patients with diffusely diseased left anterior descending coronary artery presenting to the Cardiothoracic Surgery Department in Alexandria Faculty of Medicine from January 2016 were included in this study and followed prospectively for at least 6 months. Informed consents were obtained from all patients prior to the procedure with explanation regarding the aim of the procedure and the possible side effects according to the guidelines of ethical committee at Alexandria Faculty of Medicine.

Inclusion criteria: primary elective CABG patients with diffuse LAD disease requiring endarterectomy with or without combined valvular procedure.

Exclusion criteria: patients needing endarterectomy in diffusely diseased vessels other than the LAD.

Indications for LAD endarterectomy: (1) chronic total occlusion of the LAD; (2) heavily calcific plaque impeding suturing of bypass graft to the coronary vessel; (3) multiple obstructions in the LAD; (4) diffusely diseased LAD with atherosclerosis extending into major side branches; and (5) soft atherosclerotic plaque for fear of sutures disrupting the plaque and causing distal embolization.

Surgical procedure: all procedures were done using median sternotomy and cardiopulmonary bypass. Cardioplegia was given in antegrade fashion and temperature was allowed to drift.

The most suitable soft spot was identified for LAD arteriotomy, and arteriotomy was done using super blade. Failure to pass $1 \mathrm{~mm}$ coronary probe through the arteriotomy confirmed the need for endarterectomy, which was often anticipated from the coronary angiography and other times not. The LAD arteriotomy was extended using the coronary scissors till a disease free area distal to the atherosclerotic plaque was identified 
and the plaque was removed under direct vision using the fine dissecting spatula. This method allowed complete extraction of the plaque from the LAD as well as from septal and diagonal branches if present under vision. Passage of $1 \mathrm{~mm}$ probe was done distally to ensure the distal bed was free from plaques.The proximal end of the arteriotomy was not extended beyond the proximal occlusion for fear of competitive flow between the native coronary artery and the graft. The atherosclerotic plaque was sharply divided proximally. This was followed by reconstruction using the left internal mammary artery (LIMA) or on-lay saphenous patch. LIMA was always the first choice for reconstruction. Saphenous vein patch was used in very lengthy reconstructions needing a generous patch or when harvested LIMA appeared short to avoid having a LIMA-LAD anastomosis under tension.

In cases of reconstruction using the saphenous vein, a small part of the vein graft was completely opened longitudinally using scissors to convert it to a vein patch and fashioned to match the length of the arteriotomy. The vein was then sewn to the endarterectomized LAD using prolene 7-0. A small opening was then created in the proximal or mid part of the vein patch using a scalpel and LIMA was anastomosed to the vein graft using prolene 8-0.

In cases where LIMA was used to reconstruct the LAD the LIMA was incised to match the length of the arteriotomy and it was anastomosed directly to the LAD using prolene 7-0 or 8-0 in an onlay fashion. Sometimes the surgeons prefer to start this anastomosis at the toe of the anastomosis unlike the usual where anastomosis is started at the heel. This approach makes it easier to adjust the length of the LIMA patch in case of discrepancy between the length of LIMA patch and the arteriotomy. Bulldog was removed from LIMA to allow flow in the coronary anastomosis and check if there was any leak that can be repaired before removing the cross clamp. After completion of distal anastomoses, the cross clamp was removed. Vein grafts were cut to appropriate length and proximal anastomoses to the aorta were done with partial side biting clamp.

In our study, 2 different anticoagulation protocols were used. In both regimens intravenous unfractionated heparin was started in ICU on day 0 typically after $6 \mathrm{~h}$ or as soon as chest tube output dictated with target partial thromboplastin time of 60-90 s. In the dual antiplatelet therapy (DAPT) protocol, $75 \mathrm{mg}$ clopidogrel and low dose aspirin were given on day 1 . Clopidogrel was discontinued after 1 year and aspirin given for life. The second regimen was triple therapy with warfarin for 3 months with target international normalized ratio (INR) of 2 in addition to $75 \mathrm{mg}$ clopidogrel (1 year) and low dose aspirin for life.

Echocardiography was done to all patients routinely prior to discharge. Following discharge, patients were followed up in outpatient clinic one week following discharge, then one month after discharge.

Patients enrolled in this study were contacted by phone following discharge to ask about their status regarding dyspnea and chest pain. They were asked to perform CT coronary angiography for assessment of LAD patency. CT coronary angiography was chosen for this study due to its sensitivity in assessment of bypass patency, as well as being less invasive than conventional coronary angiography.

Definitions: operative mortality was defined as death occurring during the hospitalization in which the surgery was performed or death occurring after hospital discharge, but within 30 days, unless clearly unrelated to the operation.

Stroke was defined as neurologic deficit of abrupt onset caused by disturbance in blood supply to the brain persisting $>24 \mathrm{~h}$.

Renal failure: acute or worsening renal function resulting in one or both more of the following. Increase of serum creatinine to $>2 \mathrm{mg} / \mathrm{dL}$ and to $2 \times$ the most recent preoperative creatinine or requirement for dialysis. 
Table 1. Preoperative characteristics

\begin{tabular}{lc}
\hline Preoperative characteristics & No. (\%), mean \pm SD, or median (range) $(\boldsymbol{n}=\mathbf{3 0})$ \\
\hline Age (years) & $59.23 \pm 7.98$ \\
Males & $27(90)$ \\
Cardiac profile & $26(86.7)$ \\
Unstable angina & $5(16.7)$ \\
Left main & $18(60)$ \\
Previous MI & $51.23 \pm 9.02$ \\
Ejection fraction & \\
Risk factors & $27(90)$ \\
Hypertension & $16(53.3)$ \\
Smoking & $10(33.3)$ \\
Dyslipidemia & $19(63.3)$ \\
Diabetes mellitus & $4(14.3)$ \\
Insulin use & \\
Comorbidities & $9(30)$ \\
COPD & $0(0)$ \\
Renal impairment & $1(3.3)$ \\
Previous CVA & $2(6.7)$ \\
PVD & \\
NYHA class & $15(50)$ \\
II & $15(50)$ \\
III &
\end{tabular}

MI: myocardial infarction; COPD: chronic obstructive pulmonary disease; CVA: cerebrovascular accident; PVD: peripheral vascular disease; NYHA: New York Heart Association

MI: elevation of biomarkers (creatine kinase-MB or troponin) to more than 5 times the 99th percentile of the normal reference range during the first $72 \mathrm{~h}$ after a CABG plus: (1) new pathologic $\mathrm{Q}$ waves or left bundle branch block; or (2) angiographically documented new graft or native coronary artery occlusion; or (3) imaging evidence of new loss of viable myocardium.

Statistical analyses were performed using IBM SPSS Statistics 22.0 software (IBM Corp, Armonk, NY). Normally distributed continuous variables are expressed as the mean $\pm \mathrm{SD}$, and skewed continuous variables are expressed as the median with the range. For comparison of the 2 groups, $t$ test, Wilcoxon signed rank test, or Fisher's exact test was used as deemed appropriate.

\section{RESULTS}

Baseline clinical characteristics and demographic data are summarized in Table 1. The endarterectomized LAD was reconstructed using LIMA onlay patch in 22 patients (73.3\%). Postoperative MI occurred in 2 patients, one of them only in the reconstructed LAD territory. There was one mortality occurring 5 days postoperative in the ICU due to MI and refractory ventricular arrhythmias resulting in operative mortality of $3.3 \%$. Intraoperative and postoperative data are summarized in Table 2.

At least 6 months after surgery all patients except for one case of mortality had a coronary CT angiogram done and showed 93.1 percent patency rate with 2 patients found to have stenotic LIMA-LAD anastomosis [Table 3, Figure 1]. One patient belonged to the LIMA group and the other to the saphenous patch group. In addition to the $\mathrm{CT}$ angiography echocardiogram was done and the patients were followed up clinically regarding any chest pain and dyspnea. Angina occurred in 4 patients and they belonged to Canadian Cardiovascular Society (CCS) class I. Tables 4 and 5 showed the improvement in New York Heart Association (NYHA) class and ejection fraction postoperatively.

Tables 6 and 7 showed comparison between both methods of reconstruction using LIMA or LIMA and saphenous vein patch in relation to the baseline demographics, intraoperative and postoperative events. 
Table 2. Intraoperative and postoperative data

\begin{tabular}{lll}
\hline Variables & & No. (\%), mean \pm SD, or median $($ range $(\boldsymbol{n}=\mathbf{3 0})$ \\
\hline Number of distal anastomosis & 1 & $6(20)$ \\
& 2 & $6(20)$ \\
& 3 & $12(40)$ \\
Cross clamp time (min) & 4 & $6(20)$ \\
& & $60.7 \pm 17.82$ \\
Method of reconstruction & LIMA patch & $60(28.0-100.0)$ \\
& SVG patch + LIMA & $22(73.3)$ \\
Length of reconstruction (cm) & & $8(26.7)$ \\
& & $6.35 \pm 1.47$ \\
Time of ventilation (h) & & $6(5-10)$ \\
Inotropic support (days) & $6(3-110)$ \\
ICU stay (days) & & $2.40 \pm 0.93$ \\
Myocardial infarction & $4.93 \pm 1.41$ \\
Need for dialysis & $2(6.7)$ \\
Stroke & $0(0)$ \\
Atrial fibrillation & $0(0)$ \\
Mediastinitis & $7(23.3)$ \\
Bleeding requiring exploration & $1(3.3)$ \\
Mortality & $2(6.7)$ \\
\hline
\end{tabular}

LIMA: left internal mammary artery; SVG: saphenous vein graft

Table 3. Follow up and CT angiographic patency

\begin{tabular}{ll}
\hline Variables & No. (\%), mean \pm SD, or median $($ range $)(\boldsymbol{n}=\mathbf{2 9})$ \\
\hline Duration of follow up (months) & $17.59 \pm 6.34$ \\
& $20.0(6.0-26.0)$ \\
Post operative ejection fraction & $56.28 \pm 5.62$ \\
& $58.0(48.0-68.0)$ \\
Post op. NYHA class & $20(69.0)$ \\
I & $9(31.0)$ \\
II & \\
LIMA LAD CT angio patency & $27(93.1)$ \\
Patent & $4(13.8)$ \\
Postoperative angina & $1(3.4)$ \\
Gl bleeding & $12(41.4)$ \\
Medications & $17(58.6)$ \\
Plavix aspirin & \\
Plavix aspirin + marevan &
\end{tabular}

NYHA: New York Heart Association; LIMA: left internal mammary artery; LAD: left anterior descending artery; GI: gastrointestinal

\section{DISCUSSION}

Complete revascularization of coronary vessels is the main target of CABG and in particular the LIMALAD anastomosis, since a patent LIMA-LAD is the single most important determinant of the long-term and event-free survival ${ }^{[4]}$.

With advances in PCI, patients now referred to CABG are becoming more complex with multiple comorbidities as well as less attractive target vessels. Diffuse coronary artery disease is a problem that faces surgeons now with increasing frequency since in this subset of patients PCI produces less than optimal results $^{[5]}$. In up to $25 \%$ of patients with diffuse coronary disease conventional CABG as well would not optimally revascularize the ischemic territories ${ }^{[6]}$. Therefore endarterectomy was revisited as an option to increase the surgical armamentarium in facing this complex lesion. 


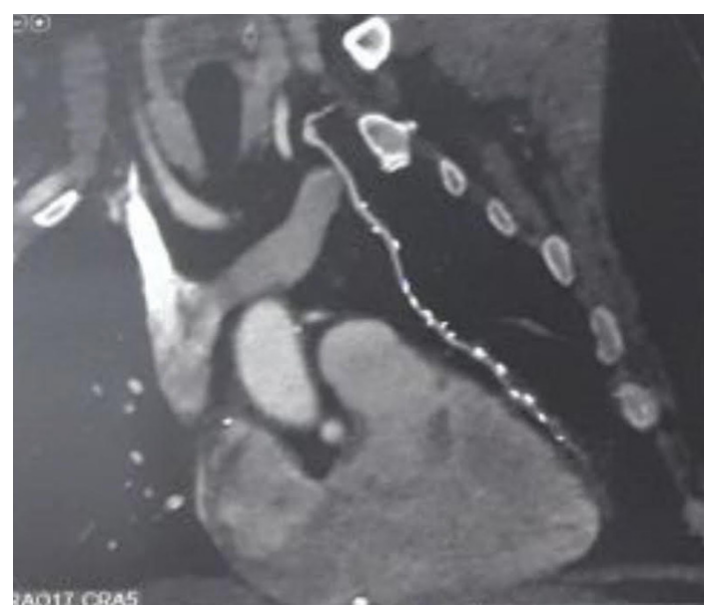

Figure 1. Representative image of CT coronary angiography showing left internal mammary artery (LIMA) patch with patent LIMA-left anterior descending artery anastomosis

Table 4. Comparison between pre and postoperative New York Heart Association class

\begin{tabular}{|c|c|c|c|c|c|c|}
\hline \multirow{2}{*}{ NYHA class } & \multicolumn{2}{|c|}{ Preoperative $(n=30)$} & \multicolumn{2}{|c|}{ Postoperative $(n=29)$} & \multirow{2}{*}{$z$} & \multirow{2}{*}{$\boldsymbol{P}$} \\
\hline & No. & $\%$ & No. & $\%$ & & \\
\hline I & 0 & 0.0 & 20 & 69.0 & & \\
\hline II & 15 & 50.0 & 9 & 31.0 & $4.919^{\star}$ & $<0.001^{\star}$ \\
\hline III & 15 & 50.0 & 0 & 0.0 & & \\
\hline
\end{tabular}

*Statistically significant at $P \leq 0.05 ; Z$ and $P$ values for Wilcoxon signed ranks test for comparing between pre and postoperative; NYHA: New York Heart Association

Table 5. Comparison between pre and postoperative ejection fraction

\begin{tabular}{|c|c|c|c|c|}
\hline & Preoperative $(n=30)$ & Postoperative $(n=29)$ & $T$ & $P$ \\
\hline \multicolumn{5}{|c|}{ Ejection fraction } \\
\hline Min-Max & $35.0-70.0$ & $48.0-68.0$ & & \\
\hline Mean \pm SD & $51.23 \pm 9.02$ & $56.28 \pm 5.62$ & $4.968^{\star}$ & $<0.001^{\star}$ \\
\hline Median & 49.0 & 58.0 & & \\
\hline
\end{tabular}

*Statistically significant at $P \leq 0.05 ; T$ and $P$ values for Paired $t$-test for comparing between pre and postoperative

Endarterectomy entails removal of the atherosclerotic core from the coronary vessel. In closed endarterectomy (also called pull out method) a small arteriotomy is used to dissect the atherosclerotic plaque out of the coronary vessel by using steady and gentle traction. It's a simpler technique but was criticized due to the possibility of occlusion of the distal LAD or its branches by insufficient endarterectomy the so called snowplow effect ${ }^{[7]}$. In addition blind traction on the atherosclerotic core can lead to tears and iatrogenic intimal flaps that would lead to occlusion of the coronary vessel ${ }^{[8]}$.

In open endarterectomy a longitudinal arteriotomy is performed on the coronary vessel beyond the limits of the atheromatous plaque and the atherosclerotic plaque is dissected under vision from the coronary vessel and its side branches avoiding any residual obstruction and repairing any intimal flaps that might occur during the removal of the plaque. The coronary is then reconstructed using an onlay patch of saphenous vein or LIMA itself. Advocates of coronary reconstruction using the saphenous vein patch suggested that it is very easy to use, easy to harvest and enlarged the lumen of the reconstructed $\operatorname{LAD}^{[6,8]}$. However others accused it of being more time consuming, as you convert one anastomosis into two anastomoses, first anastomosis of saphenous vein graft (SVG) to the endarterectomized LAD, then anastomosing the LIMA onto the saphenous vein patch. It was also suggested that this complex vascular bed formed of three 
Table 6. Relation between method of reconstruction and preoperative characteristics

\begin{tabular}{|c|c|c|c|c|}
\hline & \multicolumn{2}{|c|}{ Method of reconstruction } & \multirow{2}{*}{ Test of Sig } & \multirow{2}{*}{$\boldsymbol{P}$} \\
\hline & LIMA $(n=22)$ No. (\%) & LIMA + vein $(n=8)$ No. (\%) & & \\
\hline \multicolumn{3}{|l|}{ Gender } & \multirow{2}{*}{$\chi^{2}=2.727$} & \multirow{2}{*}{${ }^{\mathrm{FE}} P=0.166$} \\
\hline Males & $21(95.5)$ & $6(75.0)$ & & \\
\hline \multicolumn{5}{|l|}{ Age } \\
\hline Mean \pm SD & $59.4 \pm 7.69$ & $58.75 \pm 9.27$ & \multirow[t]{2}{*}{$T=0.180$} & \multirow[t]{2}{*}{0.861} \\
\hline Median & $60.0(40.0-69.0)$ & $62.0(42.0-67.0)$ & & \\
\hline \multicolumn{5}{|l|}{ Cardiac profile } \\
\hline Left main & $4(18.2)$ & $1(12.5)$ & \multirow{2}{*}{$\begin{array}{l}\chi^{2}=0.136 \\
\chi^{2}=1.285\end{array}$} & \multirow{2}{*}{$\begin{aligned}{ }^{\mathrm{FE}} P & =1.000 \\
\mathrm{FE}^{2} & =0.284\end{aligned}$} \\
\hline Unstable angina & $20(90.9) 0$ & $6(75.0)$ & & \\
\hline \multicolumn{5}{|l|}{ NYHA class } \\
\hline 1 & $0(0.0)$ & $0(0.0)$ & \multirow{3}{*}{$\chi^{2}=0.682$} & \multirow{3}{*}{${ }^{\mathrm{FE}} P=0.682$} \\
\hline$\|$ & $10(45.5)$ & $5(62.5)$ & & \\
\hline III & $12(54.5)$ & $3(37.5)$ & & \\
\hline Previous MI & $14(63.6)$ & $4(50.0)$ & $\chi^{2}=0.455$ & ${ }^{\mathrm{FE}} P=0.678$ \\
\hline \multicolumn{5}{|l|}{ Ejection fraction } \\
\hline Mean \pm SD & $50.27 \pm 8.59$ & $53.88 \pm 10.23$ & \multirow[t]{2}{*}{$T=0.967$} & \multirow[t]{2}{*}{0.342} \\
\hline Median & $48.0(35.0-70.0)$ & $54.0(40.0-70.0)$ & & \\
\hline \multicolumn{5}{|l|}{ Coronary risk factors } \\
\hline Hypertension & $19(86.4)$ & $8(100)$ & $\chi^{2}=1.212$ & ${ }^{\mathrm{FE}} P=0.545$ \\
\hline Smoking & $13(59.1)$ & $3(37.5)$ & $\chi^{2}=1.099$ & ${ }^{\mathrm{FE}} P=0.417$ \\
\hline Dyslipidemia & $5(22.7)$ & $5(62.5)$ & $\chi^{2}=4.176$ & ${ }^{\mathrm{FE}} P=0.078$ \\
\hline Diabetes mellitus & $13(59.1)$ & $6(75.0)$ & $\chi^{2}=0.639$ & ${ }^{\mathrm{FE}} P=0.672$ \\
\hline Insulin use & $3(13.6)$ & $1(12.5)$ & $\chi^{2}=0.007$ & ${ }^{\mathrm{FE}} P=1.000$ \\
\hline \multicolumn{5}{|l|}{ Comorbidities } \\
\hline COPD & $8(36.4)$ & $1(12.5)$ & $\chi^{2}=1.591$ & ${ }^{\mathrm{FE}} P=0.374$ \\
\hline Renal.impairment & $0(0.0)$ & $0(0.0)$ & - & - \\
\hline Prev.CVA & $1(4.5)$ & $0(0.0)$ & $\chi^{2}=0.376$ & ${ }^{\mathrm{FE}} P=1.000$ \\
\hline PVD & $1(4.5)$ & $1(12.5)$ & $\chi^{2}=0.597$ & ${ }^{\mathrm{FE}} P=0.469$ \\
\hline
\end{tabular}

$\chi^{2}$ : Chi square test; FE: fisher exact; T: Student $t$-test; $P$ : $P$ value for comparing between the two groups; NYHA: New York Heart Association; COPD: chronic obstructive pulmonary disease; CVA: cerebrovascular accident; PVD: peripheral vascular disease

Table 7. Comparison between both groups of reconstruction

\begin{tabular}{|c|c|c|c|c|}
\hline \multirow{2}{*}{ Variables } & \multicolumn{2}{|c|}{ Method of reconstruction } & \multirow{2}{*}{ Test of Sig } & \multirow{2}{*}{$\boldsymbol{P}$} \\
\hline & LIMA $(n=22)$ & LIMA + vein $(n=8)$ & & \\
\hline \multicolumn{5}{|l|}{ Cross clamp time (min) } \\
\hline Mean \pm SD & $58.9 \pm 18.80$ & $65.6 \pm 14.74$ & $T=0.910$ & 0.371 \\
\hline Median (range) & $60.0(28.0-100.0)$ & $70.0(40.0-85.0)$ & & \\
\hline \multicolumn{5}{|l|}{ Length of reconstruction $(\mathrm{cm})$} \\
\hline Mean \pm SD & $5.64 \pm 0.73$ & $8.31 \pm 1.16$ & $T=7.564$ & $<0.001^{\star}$ \\
\hline Median (range) & $5.50(5.0-7.0)$ & $8.0(7.0-10.0)$ & & \\
\hline \multicolumn{5}{|l|}{ Time of ventilation $(\mathrm{h})$} \\
\hline Mean \pm SD & $15.82 \pm 15.82$ & $8.0 \pm 5.50$ & $U=68.00$ & 0.344 \\
\hline Median (range) & $6.0(3.0-110.0)$ & $4.50(4.0-17.0)$ & & \\
\hline \multicolumn{5}{|l|}{ Inotropic support (days) } \\
\hline Mean \pm SD & $2.41 \pm 1.01$ & $2.38 \pm 0.74$ & $U=85.50$ & 0.889 \\
\hline Median (range) & $2.0(1.0-5.0)$ & $2.0(2.0-4.0)$ & & \\
\hline Myocardial infarction & $1(4.5)$ & $1(12.5)$ & $\chi^{2}=0.597$ & ${ }^{\mathrm{FE}} P=0.469$ \\
\hline Atrial fibrillation & $6(27.3)$ & $1(12.5)$ & $\chi^{2}=0.716$ & ${ }^{\mathrm{FE}} P=0.638$ \\
\hline Bleeding requiring reexploration & $1(4.5)$ & $1(12.5)$ & $\chi^{2}=0.597$ & ${ }^{\mathrm{FE}} P=0.469$ \\
\hline Mortality & $1(4.5)$ & $0(0.0)$ & $\chi^{2}=0.376$ & ${ }^{\mathrm{FE}} P=1.000$ \\
\hline Patency rate & $20(95.2)$ & $7(87.5)$ & $\chi^{2}=0.540$ & ${ }^{\mathrm{FE}} P=0.483$ \\
\hline Postoperative angina* & $2(9.5)$ & $2(25.0)$ & $\chi^{2}=1.167$ & ${ }^{\mathrm{FE}} P=0.300$ \\
\hline
\end{tabular}

${ }^{\star}$ One case of mortality $(n=29)$. $\chi^{2}$ : Chi square test; FE: fisher exact; $T$ : Student $t$-test; $P$ : $P$ value for comparing between the two groups; $U$ : Mann Whitney test 
components (LIMA, SVG, and native coronary) caused turbulence of flow due to difference in compliance of the the three components ${ }^{[0]}$. Enlarging the lumen of the reconstructed LAD was accused of decreasing flow velocity $^{[9]}$. In addition long term patency was questioned due to the known predilection of veins to more rapid intimal hyperplasia.

As studies continued to support the use of arterial grafting, and showing arterial grafts to show better late patency rates than SVG, the use of onlay LIMA patching to endarterectomized LAD gained wider popularity ${ }^{[10]}$. In this method the LIMA itself was used as a patch after adjusting its opening to the length of the LAD arteriotomy. Using the LIMA directly as a patch took advantage of the superior anti-atherosclerotic property of LIMA compared to SVG, besides the known vasomotor functions of the LIMA and its ability to adjust the flow rate to the distal runoff of the LAD by virtue of its release of endothelium derived relaxing factors ${ }^{[10]}$. Neverthelesss the verdict on the optimal method of LAD reconstruction is not yet clear.

Soylu et al ${ }^{[3]}$ in a best evidence series published in 2014 included 150 articles in their search and stated in conclusion that open coronary endarterectomy appeared to be safer, carried a lower rate of mortality than closed endarterectomy, and that the use of LIMA may improve mortality.

With this large study in mind and with the theoretical advantages of open over closed endarterectomy stated previously the surgeons in our study were disinclined to use the closed traction method of endarterectomy in a vessel as precious as LAD and all patients operated on in this study had open endarterectomy of the LAD.

It is worth mentioning that Barra and his colleagues from France advocated a different method of using the LIMA in reconstruction of the LAD without endarterectomy ${ }^{[11]}$. In this method LIMA onlay graft is sutured inside the coronary in such a fashion as to exclude atheromatous plaques from the lumen of the coronary artery. LIMA wall makes up $75 \%$ of the reconstructed vessel, and the newly reconstructed artery retains $25 \%$ of the native coronary artery. However this method was reserved mainly for non calcified plaques, since heavily calcific plaques can preclude suturing. They explained that by using this method they limit the use of endarterectomy and hence decrease the postoperative cascade of myofibrointimal hyperplasia and thrombosis since no area of the coronary is denuded of its covering endothelium.

Bridge or jump graft was also used for LAD reconstruction. This method was used in patients with multiple lesions in their LAD. Arteriotomies were performed proximal and distal to the site of coronary stenosis and a valveless saphenous segment was used as a bridge between the lesion to which LIMA was anastomosed ${ }^{[12]}$.

LIMA itself was used to perform jump grafts by performing a side to side anastomosis to the LAD proximal to the site of stenosis then another end to side anastomosis after jumping over the site of stenosis ${ }^{[13]}$. Again the merits of both of these methods were mainly to avoid endareterectomy of the LAD. All of the above methods were considered by the surgeons in our study for the sake of avoiding endarterectomy and its histopathological consequences and endarterectomy was saved as the last option when other methods appeared to be futile.

In our study the use of the SVG (26.7\%) was reserved for patients who needed a much lengthier reconstruction of the LAD and in cases where there was fear that the LIMA was too short or would be under tension if used in the reconstruction. This was reflected in our results showing a greater mean of reconstruction length in the saphenous vein group.

The cross clamp time was shorter in the LIMA patch group however did not reach statistical significance. Owais et al. ${ }^{[14]}$ and Myers et al. ${ }^{[15]}$ showed a statistically significant shorter cross clamp time in the LIMA patch group compared to the saphenous vein patch group. 
Two of our patients suffered from postoperative MI, one of them in the LAD region (3.3 percent). Our results compared well to rates of perioperative MI mentioned in Schmitto et al ${ }^{[16]} 3$ percent, Byrne et al. ${ }^{[17]}$ 3 percent, Myers et al. ${ }^{[15]} 4$ percent, Sundt et al. ${ }^{[18]} 2$ percent and Nishi et al. ${ }^{[19]} 2.9$ percent. There was no difference in our study between both groups LIMA and saphenous regarding MI and this was also the case in Myers et al. ${ }^{[15]}$ study.

There was one case of mortality in our study (3.3). Our explanation that the delay in starting anticoagulation for this patient who was bleeding led to a MI and started this vicious cascade of events leading to death.

Nishigawa et al.$^{[20]}$ reported mortality of 1.1 percent, Schmitto et al ${ }^{[16]} 5$ percent, Takanashi et al. ${ }^{[21]} 2.7$ percent, Byrne et al. ${ }^{[17]} 3$ percent, Nishi et al. ${ }^{[19]} 2.9$ percent. In comparing LIMA to saphenous group mortality was 4.5 percent in the LIMA group and none in the saphenous group and was not statistically significant. Myers et al. ${ }^{[15]}$ also had $4.1 \%$ mortality in the LIMA group, slightly higher than the saphenous patch group (3\%) and was not statistically significant. Owais et al ${ }^{[14]}$ had a 13 percent mortality in each of his groups, with no difference between both.

The CT coronary angiography done at 6 months postoperatively showed a patency rate of 93.1 percent, with 2 patients found to have stenotic LIMA-LAD anastomosis. One patient belonged to the LIMA group and the other to the saphenous patch group, with no statistical difference between both. Our explanation for the patient with occlusion in the LIMA and saphenous patch group was attributed to bleeding in the early postoperative hours which required reexploration in the operating room and delayed the start of the anticoagulation. The second patient had an uneventful postoperative course and this unfortunate event was not explained in his case.

In Nishigawa et al. ${ }^{[20]}$ study conventional angiography was done at a median of 7 days (range 0-85 days) and showed a patency rate of 91.6 percent. Takanashi et al. ${ }^{[21]}$ showed early patency of 94 percent.

Nishi et al. ${ }^{[19]}$ had early and late angiographic control of his patients. He had a 92.1 percent early patency rate and at midterm follow up at a mean of 21 months the patency rate was 89.1.

NYHA class significantly changed in our study following surgery, with 69 percent of patients falling in NYHA class 1 as opposed to none of the patients being in class 1 preoperatively. Schmitto et al. ${ }^{[16]}$ also noted an improvement in NYHA class after operation.

During follow up four of our patients reported recurrence of anginal pain (13.8 percent) and they belonged to CCS 1 . There was no difference related to the technique of reconstruction. Sundt $e$ a $a l^{[18]}$ reported recurrence of angina in 27 percent of their patients. Byrne et al. ${ }^{[17]}$ reported 90 percent freedom from anginal pain at 1 year. Sergeant et al. ${ }^{[22]}$ in their analysis of 9600 patients undergoing primary CABG reported recurrence of anginal pain in 8 percent of patients. Both authors attributed difference in recurrence of symptoms between patients undergoing endarterectomy and those undergoing primary grafting may be due to the diffuse and particularly severe nature of the coronary disease present among the population requiring endarterectomy and insufficient endarterectomy done in LAD or other territories.

Our anticoagulation protocol started with unfractionated heparin in the ICU. Some surgeons preferred DAPT and some triple therapy with DAPT in addition to warfarin. Takanashi et al. ${ }^{[21]}$ stated their anticoagulation protocol to be low-molecular-weight heparin, followed by triple therapy. Myers et al. ${ }^{[15]}$ used unfractionated heparin for $24 \mathrm{~h}$ and then switched to either warfarin (3 months) or clopidogrel (1 year) in addition to aspirin for life.

Nishi et al. ${ }^{[19]}$ administered low molecular weight heparin $6 \mathrm{~h}$ after arrival in the ICU followed by low-dose aspirin and warfarin $(\mathrm{INR}=2.0)$, which were continued indefinitely. 
Endarterectomy leaves behind a coronary vessel denuded of endothelium. This bare area acts as a nidus for thrombus formation and triggers a rapid coagulation cascade in the early postoperative period and can explain the high rate of postoperative MI. Therefore it seems obvious that thromboprophylaxis serves as a cornerstone in the outcome of those patients. Nevertheless anticoagulation regimens followed are based on surgeon's experience. This is due to lack of randomized controlled studies and the fact that there are no recommendations on anticoagulation and antiplatelet therapy in endarterectomy patients published in the guidelines yet.

In a recent review by Soylu et al. ${ }^{[3]}$ perioperative MI ranged from 0 to 19 percent, cerebrovascular accident ranged from 0 to 6 percent and the operative mortality ranged from 0 to 19 percent. Coronary endarterectomy was accompanied by acceptable patency rates, that ranged from $56 \%$ to $100 \%$ at a post-operative follow-up ranging from 6 months to 10 years. Our results match the results of large series in the literature.

In the current era, coronary endarterectomy appears as a valuable surgical option in diffusely diseased coronary vessels. With appropriate indications and in experienced hands, it can be done with acceptable morbidity, mortality, angiographic patency rates and lead to favorable outcomes in a high risk cohort.

In conclusion, results of reconstruction by LIMA and saphenous vein patch are comparable in short term follow up.

\section{DECLARATIONS}

\section{Acknowledgments}

Dr. Bassem Ramadan for sharing his expertise in coronary reconstruction.

\section{Authors' contributions}

Collection of data, analysis, follow up of patients, writing up the manuscript: Elsayed $\mathrm{MH}$

Design of the study and revision, operated most of the cases: Hassanein WM

Revision of the manuscript: Keshk SA, Etman WG

Follow up of cases: Zidan M

\section{Availability of data and materials}

Alexandria University Cardiothoracic Surgery Department database.

\section{Financial support and sponsorship}

None.

\section{Conflicts of interest}

All authors declared that there are no conflicts of interest.

\section{Ethical approval and consent to participate}

Informed consents were obtained from all patients prior to the procedure with explanation regarding the aim of the procedure and the possible side effects according to the guidelines of ethical committee at Alexandria Faculty of Medicine.

\section{Consent for publication}

Not applicable.

\section{Copyright}

(c) The Author(s) 2018. 


\section{REFERENCES}

1. Garcia S, Sandoval Y, Roukoz H, Adabag S, Canoniero M, et al. Outcomes after complete versus incomplete revascularization of patients with multivessel coronary artery disease: a meta-analysis of 89,883 patients enrolled in randomized clinical trials and observational studies. J Am Coll Cardiol 2013;62:1421-31.

2. Bailey CP, May A, Lemmon WM. Survival after coronary endarterectomy in man. J Am Med Assoc 1957;164:641-6.

3. Soylu E, Harling L, Ashrafian H, Athanasiou T. Does coronary endarterectomy technique affect surgical outcome when combined with coronary artery bypass grafting? Interact Cardiovasc Thorac Surg 2014;19:848-55.

4. Lytle BW, Loop FD, Cosgrove DM, Ratliff NB, Easley K, et al. Longterm (5 to 12 years) serial studies of internal mammary artery and saphenous vein coronary bypass grafts. J Thorac Cardiovasc Surg 1985;89:248-58.

5. Baranauskas A, Peace A, Kibarskis A, Shannon J, Abraitis V, et al. FFR result post PCI is suboptimal in long diffuse coronary artery disease. Eurointervention 2016;12:1473-80.

6. Santini F, Casali G, Lusini M, D’Onofrio A, Barbieri E, et al. Mid-term results after extensive vein patch reconstruction and internal mammary grafting of the diffusely diseased left anterior descending coronary artery. Eur J Cardiothorac Surg 2002;21:1020-5.

7. Shapira N, Lumia FJ, Gottdiener JS, Germon P, Lemole GM. Adjunct endarterectomy of the left anterior descending coronary artery. Ann Thorac Surg 1988;46:289-96.

8. Fundarò P, Di Biasi P, Santoli C. Coronary endarterectomy combined with vein patch reconstruction and internal mammary artery grafting: experience with 18 patients. Texas Heart Inst J 1987;14:389-94.

9. FitzGibbon GM, Leach AJ, Keon WJ, Burton JR, Kafka HP. Coronary bypass graft fate. Angiographic study of 1,179 vein grafts early, one year, and five years after operation. J Thorac Cardiovasc Surg 1986;91:773-8.

10. Lüscher TF, Diederich D, Siebenmann R, Lehmann K, Stulz P, et al. Difference between endothelium-dependent relaxation in arterial and in venous coronary bypass grafts. N Engl J Med 1988;319:462-7.

11. Barra JA, Bezon E, Mondine P, Resk A, Gilard M, et al. Coronary artery reconstruction for extensive coronary disease: 108 patients and two year follow-up. Ann Thorac Surg 2000;70:1541-5.

12. Gucu A, Goncu T, Yavuz S, Ozluk OA, Eris C, et al. Alternative option in patients with multisegmental left anterior descending coronary artery disease for providing complete myocardial revascularization. Int J Clin Exp Med 2014;7:142-7.

13. Nezic D, Knezevic A, Milojevic P, Jovic M, Sagic D, et al. Tandem pedicled internal thoracic artery conduit for sequential grafting of multiple left anterior descending coronary artery lesions. Tex Heart Inst J 2006;33:469-72.

14. Owais T, Abdelfattah I, Osama A, Rasekh F, Girdauskas E, et al. Early term results of left internal mammary artery patch to left anterior descending artery and left internal mammary artery to on-lay saphenous vein patch in diffusely diseased left anterior descending artery: which is inferior and which is superior? Int J Cardiovasc Res 2016;5:3.

15. Myers PO, Tabata M, Shekar PS, Couper GS, Khalpey ZI, et al. Extensive endarterectomy and reconstruction of the left anterior descending artery: early and late outcomes. J Thorac Cardiovasc Surg 2012;143:1336-40.

16. Schmitto JD, Kolat P, Ortmann P, Popov AF, Coskun KO, et al. Early results of coronary artery bypass grafting with coronary endarterectomy for severe coronary artery disease. J Cardiothorac Surg 2009;4:52.

17. Byrne JG, Karavas AN, Gudbjartson T, Leacche M, Rawn JD, et al. Left anterior descending coronary endarterectomy: early and late results in 196 consecutive patients. Ann Thorac Surg 2004;78:867-73.

18. Sundt TM 3rd, Camillo CJ, Mendeloff EN, Barner HB, Gay WA Jr. Reappraisal of coronary endarterectomy for the treatment of diffuse coronary artery disease. Ann Thorac Surg 1999;68:1272-7.

19. Nishi H, Miyamoto S, Takanashi S, Minamimura H, Ishikawa T, et al. Optimal method of coronary endarterectomy for diffusely diseased coronary arteries. Ann Thorac Surg 2005;79:846-53.

20. Nishigawa K, Fukui T, Yamazaki M, Takanashi S. Ten-year experience of coronary endarterectomy for the diffusely diseased left anterior descending artery. Ann Thorac Surg 2017;103:710-6.

21. Takanashi S, Fukui T, Miyamoto Y. Coronary endarterectomy in the left anterior descending artery. J Cardiol 2008;52:261-8.

22. Sergeant P, Blackstone E, Meyns B. Is return of angina after coronary artery bypass grafting immutable, can it be delayed, and is it important? J Thorac Cardiovasc Surg 1998;116:440-53. 